\title{
ON THE PERIODIC MOTIONS OF DYNAMICAL SYSTEMS.
}

\author{
By
}

GEORGE D. BIRKHOFF

of Cambridge, U. S: A.

\section{Introduction.}

In his work on dynamies Poincaré was led to focus attention primarily upon the periodic motions. He conjectured that any motion of a dynamical system might be approximated by means of those of periodic type, i.e. that the periodic motions would be found to be densely distributed among all possible motions; and it became a task of the first order of importance for him to determine what the actual distribution of the periodic motions was, so as to prove or disprove his conjecture.

Poincaré employed the method of analytic continuation in his great Prize Memoir in the Acta Mathematica, which dealt with the problem of $n$ bodies. In the integrable limiting case when the masses of all but one of the bodies vanish, there are infinitely many periodic motions. By varying certain parameters he passed from this trivial limiting case to the case when none of the masses are zero, and showed that these periodic motions persist as members of analytic families, unless two of them combine and disappear from the real domain like the roots of algebraic equations with real coefficients. He did not consider the possibility of disappearance of such a motion by its period becoming infinite, although this possibility requires consideration also.

Unfortunately this method of analytic continuation gave very meagre results, for the following reason. Although there are infinitely many periodic families, it is conceivable that the range of the parameters becomes less and less as the type of the periodic motion becomes more and more complicated: 
This would mean that only a finite number of the periodic motions might exist for any particular set of the values of the parameters other than that of the trivial integrable case.

Thus Poincare found the method of analytic continuation to be insufficient, and was forced to seek other instruments of attack. To begin with, he fastened attention mainly upon the simplest possible case with two degrees of freedom, namely the so-called restricted problem of three bodies. Almost all of the qualitative reasoning in his Méthodes nouvelles de la Mécanique céleste deals only with this case.

Notwithstanding this severe limitation, and despite many years of effort, Poincaré was not able fully to attain his goal. Near the end of his life he gave out his last geometric theorem without complete proof. ${ }^{1}$ By its means he showed that in the restricted problem of three bodies and analogous problems, an infinite number of periodic motions would exist. In a recent paper ${ }^{2}$ I have generalized this theorem and my earlier proof of it, although without giving the dynamical application. At the kind invitation of Professor Mittag-Leffler, I endeavour to set forth here, with as little technicality as possible, the essential facts known to me concerning the distribution of the periodic motions, particularly as based on an application of the geometric theorem of Poincaré and its generalization.

\section{The billiard ball on a convex table.}

In order to see how the theorem of Poincaré and its generalization can be applied to dynamical systems with two degrees of freedom, I propose to draw attention to a special but highly typical system of this sort, namely that afforded by the motion of a billiard ball upon a convex billiard table (Fig. I). This example is very illuminating for the following reason: Any dynamical system with two degrees of freedom is isomorphic with the motion of a particle on a smooth surface rotating uniformly about a fixed axis and carrying a conservative field of force with it. ${ }^{3}$ In particular if the surface is not rotating and if the field of force is lacking, the paths of the particles will be geodesics. If the surface

1. Sur un théorème de Géométrie, Rendiconti del Circolo Matematico di Palermo, vol. 33, 1912.

2 An Extension of Poincarë's Last Geometric Theorem, Acta Mathematica, vol. 47, 1926.

${ }^{3}$ See my paper, "Dynamical Systems With Tuo Degrees of Freedom", Transactions of the American Mathematical Society, vol. 18, 1917. It is assumed that the Lagrangian principal funetion $L$ is quadratic in the velocities. 
is conceived of as convex to begin with and then gradually to be flattened to the form of a plane convex curve $C$, the »billiard ball» problem results. But in this problem the formal side, usually so formidable in dynamics, almost completely disappears, and only the interesting qualitative questions need to be considered. If $C$ happens to be an ellipse an integrable system results, namely as a limiting case of the geodesics on an ellipsoid treated by Jacobi.

In this problem one can arrive at the existence of certain periodic motions by direct maximum-minimum methods. As of interest in itself $I$ wish to show how this can be done. Results which are being obtained by Morse (but not yet published) indicate that the scope of these methods, already developed to some extent by Hadamard, Poincaré, Whittaker and myself, can be further extended.

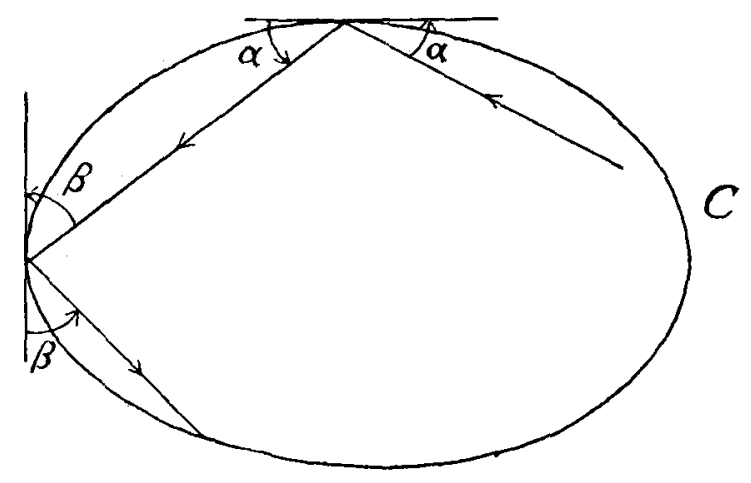

Fig. 1.

Thus the power of such maximum-minimum considerations in the billiard ball problem is likely to prove typical of the general case.

Any longest chord of the curve $C$ (or boundary of the billiard table) when traversed in both directions evidently yields one of the simplest periodic motions. The billiard ball moving along this chord strikes the curved boundary at right angles and recoils along it in the opposite direction. If we seek to vary this chord continuously, while diminishing its length as little as possible, so as finally to interchange its two ends, there will be an intermediate position of least length which will be the chord $C$ where $C$ is of least breadth. Detailed computation of the slightly perturbed motions indicates that the first of these two periodic motions is unstable, while the second is stable, i.e. with formal trigonometric series for the perturbations.

Next we ask for the triangle of maximum length inscribed in $C$. Evidently at least one such triangle will exist, and can have no degenerate side of zero 46-26404. Acta mathematica. 50. Imprimé le 27 octobre 1927. 
length. At each of its vertices the tangent will, of course, make equal angles with the two sides passing through the vertex. Hence a harmonic triangle is obtained which will correspond to two distinct motions, one for each of the two possible senses of description.

Moreover if we seek to vary this triangle continuously, without changing the order of its vertices and diminishing the perimeter as little as possible, so as finally to advance the vertices cyclically, we discover a second harmonic triangle, also corresponding to two periodic motions.

In this way the existence of two harmonic $n$ sided polygons which make $k$ circuits of the curve $C$ ( $k$ less than $\frac{n}{2}$ and prime to $\left.n\right)$ can be proved. The two motions corresponding to the polygon of maximum type will be unstable, while the other of minimax type may be stable or unstable.

In the case of a circular boundary the totality of regular inscribed polygons (simple or cross) form the harmonic polygons.

We propose next to set up a ring transformation associated with the billiard ball problem, and to show how the geometric theorem of Poincaré in its first form leads to the facts deduced above. The reduction to a ring transformation is of fundamental theoretic importance, quite aside from the relation to the question of periodic motions. It should be noted also that in the cases of most interest like the restricted problem of three bodies ${ }^{1}$ the method of reduction to a ring transformation and application of the theorem of Poincare is available for the treatment of the periodic motions, while the method of maximum-minimum has not as yet been shown to be applicable.

\section{Reduction to a ring transformation $T$.}

To begin with we suppose the length of $C$ to be $2 \pi$ and to be measured from a fixed point $O$ to a variable point $P$ by an angular coordinate $\varphi$ (Fig. 2).

At $P$, taken as the point of projection of the billiard ball, let $\theta$ denote the angle between the positive direction of the tangent and the direction of projection. The variable $\theta$ varies between $O$ and $\pi$ only. These coordinates $\theta, \varphi$ suffice to represent all possible states of projection unambiguously. If $\varphi$ be taken as an angular coordinate in the plane, while $\theta$, angmented by a constant, say $\pi$, be taken as a radial coordinate, the set of values $\theta, \varphi$ are represented on a ring

${ }^{1}$ See my paper On the Restricted Problem of Three Bodies, Rendiconti del Circolo Matematico di Palermo, vol. 39, I9I5, and the paper of Poincare cited above. 
bounded by concentric circles of radius $\pi$ and $2 \pi$ respectively, namely the circles $\theta=0$ and $\theta=\pi$ (Fig. 3).

Consider now a definite state of projection at $P$ with given $\theta, \varphi$. The billiard ball leaves the edge at $P$ to strike it again at $P_{1}$, there to be projected in a state $\theta_{1}, \varphi_{1}$, say, and so forth indefinitely. If $C$ is an analytic curve, as we assume it to be, the correspondence between $\theta, \varphi$ and $\theta_{1}, \varphi_{1}$ is evidently one-toone and analytic within the ring. When $\theta$ is nearly $\mathrm{o}$ or $\pi$, the ball is projected at a slight angle to the edge, and strikes it again at a nearby॰ point with $\theta$ nearly $O$ or $\pi$ as the case may be. Hence the points on the bounding circles correspond to themselves. with $\theta_{1}=\theta, \varphi_{1}=\varphi$.

One further remark needs to be made about the correspondence along the two boundaries of the ring. If we think of each point $(\theta, \varphi)$ as being carried

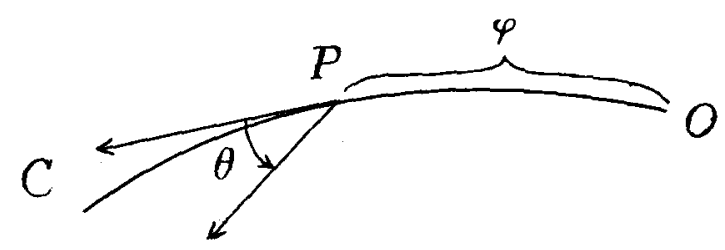

Fig. 2.

into $\left(\theta_{1}, \varphi_{1}\right)$ by a transformation or deformation of the ring, this transformation $T$ will effect a certain number of complete rotations of the inner circle, and also of the outer circle, since the points of these boundaries are invariant as just seen. We may arbitrarily regard the inner circle as having undergone no rotation, but the same will not then be true of the outer circle which can at once be shown to have undergone a single complete revolution in the positive sense. For let the projection angle $\theta$ for a given point $P$ with corresponding fixed $\varphi$ vary from o to $\pi$. It is obvious that then $\theta_{1}$ will increase from o to $\pi$ while $\varphi$ increases by $2 \pi$ since the point $P_{1}$ makes a complete circuit of $C$ in a positive sense. In other words, the transformation $T$ takes radial segments across the ring into curves starting at the same point of the inner circle but winding around the ring just once while crossing it. Hence the outer boundary has undergone a single positive revolution under the transformation $T$.

Suppose now that we have a periodic motion, for example that corresponding to one of the harmonic triangles taken in a positive sense. It is evident that the transformation $T$ of the ring takes the point of the ring representing the state of projection at the first vertex into that of the second; and likewise takes the state for the second vertex into that for the third, and that for the 
third vertex into the first. Thus when $T$ is applied, the triple of points on the ring is cyclically advanced, and each point of the triple is unaltered by the application of the third iterate $T^{3}$ of $T$.

Conversely to any triple with this property, or to any point invariant under $T^{3}$ together with its images under $T$ and $T^{2}$, corresponds a motion belonging to a harmonic triangle. Evidently then from considerations advanced earlier there are at least four such triples.

It is obvious that there can be no invariant points under $T$ itself, because $\varphi$ is increased but by less than $2 \pi$.

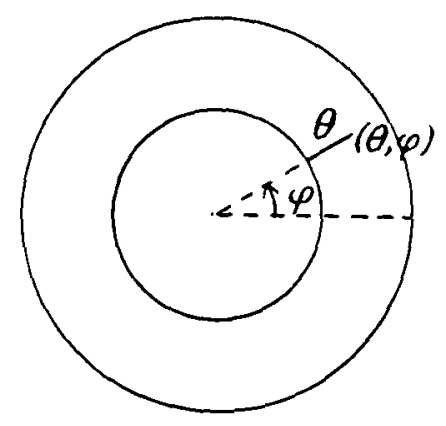

Fig. 3.

In this way the search for harmonic polygons and the allied periodic motions in the billiard ball problem resolves itself into the determination of sets of distinct points $P_{1}, \ldots P_{n}$ cyclically advanced by $T$, so that in general we have $T^{n}\left(P_{i}\right)=P_{i}(i=\mathrm{I}, \ldots n)$.

It could be shown more generally that each and every interesting property of the motion of the billiard ball is mirrored in a corresponding property of the transformation $T$. Thus the dynamical problem is effectively reduced to that of a particular transformation of a circular ring into itself.

\section{The invariant integral.}

There is a further property of the transformation

$$
T: \theta_{1}=f(\theta, \varphi), \varphi_{1}=g(\theta, \varphi)
$$

which plays a fundamental part in applying the geometric theorem of Poincaré: the double integral $\iint \sin \theta d \theta d \varphi$ taken over any area $\sigma$ of the ring has the same value as over the images $\sigma_{1}, \sigma_{2}, \ldots$ under $T$ and its iterates. 
Before passing to the entirely elementary proof of this fact, one immediate conclusion may be cited in justification of the statement as to the fundamental theoretic importance of the ring transformation which was made earlier. Since the integrals evaluated over $\sigma, \sigma_{1}, \sigma_{2}, \ldots$ have the same value, and since its value over the entire ring is finite, being $4 \pi$, some two of the images $\sigma_{i}$ and $\sigma_{j}$ overlap. Employing the inverse transformation we infer that $\sigma_{i-1}$ and $\sigma_{j-1}$ also overlap, and thus finally that $\sigma_{i-j}$ and $\sigma$ overlap $(i>j)$. But, interpreted for the billiard ball problem, this means that the ball can be projected very nearly, with arbitrary position and direction, to return subsequently to nearly the same position and direction. As elaborated by Poincaré, this chain of reasoning leads to the conclusion that the "probability" is unity for an arbitrary motion to return infinitely often to the neighborhood of its initial state. He called this property of the dynamical system "stability in the sense of Poisson".

The proof that the double integral is invariant depends on an explicit evaluation of the Jacobian

$$
J=\frac{\partial \theta_{1}}{\partial \theta} \frac{\partial \varphi_{1}}{\partial \varphi}-\frac{\partial \theta_{1}}{\partial \varphi} \frac{\partial \varphi_{1}}{\partial \theta}
$$

In fact if $\iint M(\theta, \varphi) d \theta d \varphi$ is invariant, we have

$$
\iint_{\sigma_{1}} M\left(\theta_{1}, \varphi_{1}\right) d \theta_{1} d \varphi_{1}=\iint_{\sigma} M(\theta, \varphi) d \theta d \varphi
$$

where the variables $\theta_{1}, \varphi_{1}$ range over the region $\sigma_{1}$ just as $\theta, \varphi$ do over $\sigma$. But according to the fundamental theorem for change of variables, $T$ gives the integral on the left the form

$$
\iint_{\sigma} M\left(\theta_{1}, \varphi_{1}\right) J d \theta d \varphi
$$

Comparing this expression and the integral on the right which are both integrals over the same arbitrary region $\sigma$ we deduce the functional relation:

$$
M\left(\theta_{1}, \varphi_{1}\right) J=M(\theta, \varphi)
$$

as the well known necessary and also sufficient condition for invariance. Hence to establish that $\iint \sin \theta d \theta d \varphi$ is invariant we must only prove 


$$
J=\frac{\sin \theta}{\sin \theta_{1}}
$$

Let

$$
x=F(\varphi), y=G(\varphi)
$$

be the equations of $C$ in rectangular coordinates, so that, if $\tau$ denotes the angle between the positive tangential direction at a point of $C$ and the positive $x$ axis, we have

$$
\tau=\tan ^{-1} \frac{G^{\prime}(\varphi)}{F^{\prime}(\varphi)}
$$

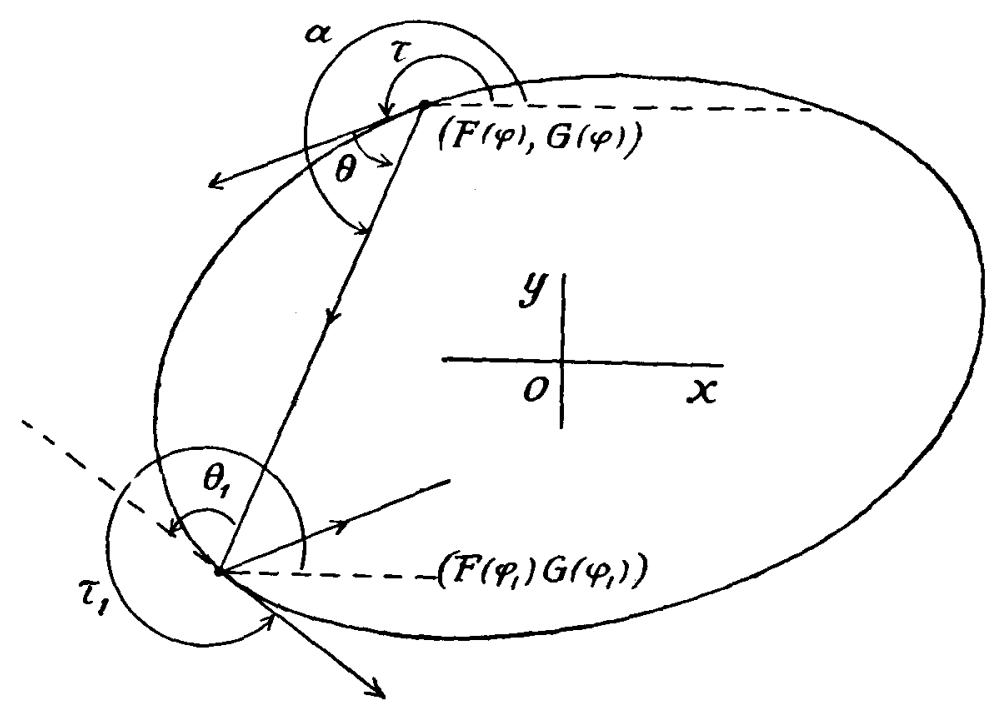

Fig. 4.

Similary let $\tau_{1}$ denote the like angle at the transformed point, which will be given by the same expression save that $\varphi$ is replaced by $\varphi_{1}$. Finally let $\alpha$ designate the angle between the positively directed $x$ axis and the direction of initial projection (Fig. 4). It is evident that the following two relations will hold

$$
\theta=\alpha-\tau, \theta_{1}=\tau_{1}-\alpha
$$

Substituting in the above value for $\tau$ and the analogous value for $\tau_{1}$, and also substituting in for $\alpha$ the value

$$
\tan ^{-1} \frac{G\left(\varphi_{1}\right)-G(\varphi)}{F\left(\varphi_{1}\right)-F(\varphi)}
$$


evident by inspection, we obtain the explicit formulas

$$
T:\left\{\begin{array}{l}
\theta=\tan ^{-1} \frac{G\left(\varphi_{1}\right)-G(\varphi)}{F\left(\varphi_{1}\right)-F(\varphi)}-\tan ^{-1} \frac{G^{\prime}(\varphi)}{F^{\prime}(\varphi)} \equiv L\left(\dot{\varphi}, \varphi_{1}\right), \\
\theta_{1}=\tan ^{-1} \frac{G^{\prime}\left(\varphi_{1}\right)}{F^{\prime}\left(\varphi_{1}\right)}-\tan ^{-1} \frac{G\left(\varphi_{1}\right)-G(\varphi)}{F\left(\varphi_{1}\right)-F(\varphi)} \equiv M\left(\varphi, \varphi_{1}\right) .
\end{array}\right.
$$

These two equations define the transformation $T$ from $(\theta, \varphi)$ to $\left(\theta_{1}, \varphi_{1}\right)$.

Taking differentials we find

$$
d \theta=L_{\varphi} d \varphi+L_{\varphi_{\mathrm{t}}} d \varphi_{1}, d \theta_{1}=M_{\varphi} d \varphi+M_{\varphi_{1}} d \varphi_{1}
$$

whence at once

$$
\left\{\begin{array}{l}
d \theta_{1}=\frac{M_{\varphi_{1}}}{L_{\varphi_{1}}} d \theta+\left(M_{\varphi}-\frac{M_{\varphi_{1}} L_{\varphi}}{L_{\varphi_{1}}}\right) d \varphi \\
d \varphi_{1}=\frac{\mathrm{I}}{L_{\varphi_{1}}} d \theta-\frac{L_{\varphi}}{L_{\varphi_{1}}} d \varphi
\end{array}\right.
$$

This gives us the Jacobian

$$
J=-\frac{M_{\varphi}}{L_{\varphi_{1}}}=-\frac{\left[F\left(\varphi_{1}\right)-F(\varphi)\right] G^{\prime}(\varphi)-\left[G\left(\varphi_{1}\right)-G(\varphi)\right] F^{\prime}(\varphi)}{\left[F\left(\varphi_{1}\right)-F(\varphi)\right] G^{\prime}\left(\varphi_{1}\right)-\left[G\left(\varphi_{1}\right)-G(\varphi)\right] F^{\prime}\left(\varphi_{1}\right)}
$$

But $F\left(\varphi_{1}\right)-F(\varphi), G\left(\varphi_{1}\right)-G(\varphi)$ are proportional to $\cos \alpha, \sin \alpha$ respectively, while we have also

$$
F^{\prime}(\varphi)==\cos \tau, G^{\prime}(\varphi)=\sin \tau, F^{\prime}\left(\varphi_{1}\right)=\cos \tau_{1}, G^{\prime}\left(\varphi_{1}\right)=\sin \tau_{1}
$$

so that finally we obtain

$$
J=\frac{\sin (\alpha-\tau)}{\sin \left(\tau_{1}-\alpha\right)}=\frac{\sin \theta}{\sin \theta_{1}}
$$

as was stated.

\section{Application of the theorem of Poincaré.}

As has been seen, there are no points of the ring which are invariant under $T$. On the other hand consider $T^{2}$ followed by a rotation of the $\theta, \varphi$ plane through an angle $-2 \pi$ which we designate by $R_{-1}$. The resultant transformation of the ring admits the same area integral as $T$, of course, but advances the points of the outer circle by an angle $2 \pi$, and those of the inner circle by an angle 
$-2 \pi$ of opposite sign. These are the two conditions essential for the application of the theorem which states that any one-to-one transformation of a ring, admitting an invariant integral and rotating the boundaries in opposite angular directions, possesses at least two distinct invariant points with indices of opposite signs. Hence $R_{-1} T^{2}$ (the compound transformation) possesses two such invariant points. This means that $T^{2}$ has two geometrically distinct invariant points of oppositely signed indices ${ }^{1}$, although these correspond to an increase of $2 \pi$ for $\varphi$.

If $P$ is such an invariant point, so is $T(P)$ of course but with the same index. Thus we get two point pairs, say

$$
P, T(P) ; Q, T(Q)
$$

all four distinct. These evidently correspond to the two fundamental periodic motions.

For the application of the theorem of Poincare to the periodic motions of more complicated type it is necessary to take account of the fact that every such motion is associated with a distinct second such motion obtained by reversing the direction of motion, although these motions have the same index. However, one of these motions increases $\varphi$ by $2 k \pi$ while the other increases it by $2(n-k) \pi$. By only considering invariant points of $T^{n}(n>2)$ for which $\varphi$ increases by $2 k \pi, k \leqq \frac{n}{2}$ we clearly obtain each harmonic $n$ sided polygon only once. It may be noted in passing that this pairing of motions in the billiard ball problem is fully reflected in the fact that $T$ is a product of two involutory transformations: it was the same special property of the ring transformation in the restricted problem of three bodies which enabled me to prove the existance of infinitely many symmetric periodic orbits. ${ }^{2}$

Now turn to the invariant points of the compound transformation $R_{-k} T^{n}$ where $R_{k}$ denotes a $k$ fold rotation through the angle $-2 \pi$. The rotations on the outer and inner circles are clearly

$$
2(n-k) \pi \text { and } 2 k \pi,
$$

which will be of opposite sign if $0<k<\frac{n}{2}$. Thence we can infer the existence of at least two geometrically distinct series of points

${ }^{1}$ See my recent Acta article (loc. eit.). By the index of an invariant point is meant the total changes in angular direction of a line joining a point $P$ to its image $P_{1}$ when $P$ makes a small positive circuit of the invariant point.

${ }^{2}$ See my paper in the Rendiconti di Palermo, loe. cit. 


$$
\left\{\begin{array}{l}
P, T(P), \ldots T^{n-1}(P) \\
Q, T(Q), \ldots T^{n-1}(Q)
\end{array}\right.
$$

such that we have $T^{n}(P)==P, T^{n}(Q)=Q$ while $\varphi$ has been increased by $2 k x$; it is assumed that $k$ and $n$ are relatively prime.

To prove this assertion in detail, we may let $P$ be one such invariant point, such that $T^{n}$ increases $\varphi$ by $2 k \pi$. If

$$
P, T(P), \ldots T^{n-1}(P)
$$

are not distinct, let $T^{m}(P)=P(m<n-1)$ and suppose that $\varphi$ is increased by $2 j \pi$. By combination of the two symbolic equations $T^{m}(P)=P, T^{n}(P)=P$ we obtain $T^{d}(P)=P$ where $d\left(\neq_{\mathrm{I}}\right)$ is the greatest common divisor of $m$ and $n$. Thus $P$ is invariant under $T^{d}$. Suppose that under $T^{d}$ the $\varphi$ of $P$ increases by $2 f \pi$. From the equation $n=q d$ we see that $T^{n}$ will then increase the $\varphi$ of $P$ by $2 q f \pi$ so that $k=q f$. Thus $k$ and $n$ would possess a common factor, contrary to hypothesis.

Also not only are the first series of $n$ points distinct but these have the same index. Hence there will be a point $Q$ invariant of $T^{n}$ and with oppositely signed index. This with its images under successive powers of $T$ will necessarily be distinct from the closed set generated by $P$, and leads to a second distinct set generated by $Q$.

Hence we obtain for every $n$ and every relatively prime $k<\frac{n}{2}$ two geometrically distinct harmonic polygons with $n$ sides and making $k$ circuits of the curve $C$. Corresponding to these there will be, of course, four periodic motions. We shall not attempt to develop here the characteristics as to type of stability and instability dependent upon the sign of the index.

It is worth while to point out the general significance of such ring transformations for dynamical systems with two degree of freedom. For such a system there are two space coordinates $p, q$ and two velocity coordinates $p^{\prime}, q^{\prime}$. These four quantities determine a state of motion, or a 'point' in the four dimensional manifold of states of motion. Each motion is represented as a curve in the manifold of states of motion. But the energy integral shows that these curves lie on known three-dimensional sub-manifolds, upon one of which we fix attention. Suppose that we can find a two-dimensional ring in the submanifold, which is bounded by two closed curves of motion and which is cut by all the other curves of motion infinitely often and in the same sense. A point $P$ of the ring, followed along its curve of motion in a positive sense till it cuts 47-26404. Acta mathematica. 50. Imprimé le 27 octobre 1927. 
the ring again at $P_{1}$, defines a transformation $T$ of the ring into itself, nanely the transformation which takes $P$ into its image $P_{1}$. This transformation $T$ and its powers will in general possess the properties necessary for the aplication of Poincaré's last geometric theorem, and thus there must exist infinitely many further periodic motions. Unfortunately such a ring is not known to exist in general, although it does in some interesting cases. Furthermore it will be readily believed that the analytic labor of actually setting up the ring, and the transformation, is large. In the billiard ball problem, the integral used is that of constant energy, while the auxiliary periodic motions evidently are those obtained by a rolling motion of the billiard ball around the ring in either sense.

\section{Application of the generalized theorem.}

For the application of the geometric theorem of Poincaré used above it is essential to have two stable periodic motions corresponding to the boundaries of the ring, and then to obtain the ring itself in case it exists.

The generalized theorem differs essentially from the form so far employed here in that only one boundary of the ring, say the inner, is required to be invariant under the transformation. However, it is required that the image of the outer circle under $T$ be cut only once by any radius vector, and that its points be angularly advanced in the opposite sense from that of the inner circle. To apply the theorem it is only necessary to know a single stable periodic motion. The conclusion to be drawn from it is that there exist two and hence infinitely many other periodic motions in the immediate neighborhood of the given stable periodic motion. Thus every stable periodic motion is a cluster motion for infinitely many other periodic motions near to it, but in general making many circuits about it before reentering.

We shall make use of the billiard ball problem again in applying the generalized theorem, although obviously the method is entirely general. It does not seem evident that the same results can be obtained by the maximum-minimum method. In fact the success in application depends on details which do not seem to come into play in the use of maximum-minimum considerations.

A typical stable periodic orbit with which to start is the simplest one of stable type which traces out twice the chord crossing $C$ where it is of least breadth. To it there corresponds an invariant point under $T^{2}$ of stable type. Considerations based only upon the existence of an invariant area integral show that for suitably taken coordinates, the transformation $T$ may be given the form: 


$$
\left\{\begin{array}{l}
u_{1}=u \cos \left(\psi_{0}-c r^{2}\right)-v \sin \left(\psi_{0}-c r^{2}\right)+P_{n}(u, v) \\
v_{1}=u \sin \left(\psi_{0}-c r^{2}\right)+v \cos \left(\psi_{0}-c r^{2}\right)+Q_{n}(u, v)
\end{array}\right.
$$

near the invariant point taken at $(0,0){ }^{1}$ Here $r^{2}$ stands for $u^{2}+v^{2}$ while $P_{n}, Q_{n}$ are convergent power series in $u, v$ beginning with terms of the $n$th degree in $u, v$, with $n$ arbitrarily large. The constant $\psi_{0}$ is supposed to be incommensurable with $2 \pi$, and $c$ to be different from $o$. Both of these conditions are in general satisfied. When they fail to be satisfied although the motion be of stable type, the generalised theorem remains applicable, but this fact cannot be touched upon here.

From the form of this transformation it is apparent that the circles $r^{2}=k$ are carried into curves

$$
r^{2}+R_{n}(u, v)=k
$$

where $R_{n}$ is of the $n$th order in $u, v$. Such a curve will differ only very slightly from the circle from which it came. In general it is apparent that in polar coordinates the transformation $T$ is very closely like the following:

$$
r_{1}=r, \psi_{1}=\psi+\psi_{0}-c r^{2}
$$

near the origin. But the $n$th power of this transformation rotates the radial directions at the invariant point by $n \psi_{0}$ and on the circle $r=\varrho_{0}$ by $n\left(\psi_{0}-c \varrho_{0}{ }^{2}\right)$. Hence if $n$ is so large that for some integer $k$

$$
n\left(\psi_{0}-c \varrho_{0}{ }^{2}\right)<2 k \pi<n \psi_{0}
$$

the original theorem of Poincaré would be applicable, with the ring bounded by the circles $r=0$ and $r=\varrho_{0}$, and with the transformation $R_{-k} \bar{T}^{n}$ where $R_{-k}$ stands for a rotation through an angle $-2 k \pi$, and $\bar{T}$ for the transformation written explicitly above. Of course this ring may be expanded radially by a constant distance so as to yield the usual form of ring transformation.

Now it may be established that for suitably taken $k$ and $n$ the approximation of the actual transformation $R_{-k} T^{n}$ is so close that the outer boundary, although not the original circle $r=\varrho_{0}$, is a curve met once and only once by any

'See my earlier article Surface Transformations and Their Dynamical Applications, Acta Mathematica, vol. 43, 1922. 
radius vector, while the inner and outer boundaries are still advanced in opposite sences. The details cannot of course be given here.

Thus the generalized theorem shows that there exist two invariant points of $T^{n}$ within the circle $r=\varrho_{0}$, which advance $\varphi$ by $2 k \pi$ in an angular sense. Evidently the periodic motions so obtained are uniformly near to the stable motion which we began with. In this way we can infer the existence of infinitely many periodic motions in the immediate neighborhood of the simplest periodic motion of stable type, so that there are infinitely many harmonic polygons lying in the immediate neighborhood of the chord crossing the curve $C$ at its narrowest part. A more careful examination of the asymptotic form of the transformation $T$ near the invariant point shows that such further invariant points obtained with positive index are stable, while those of negative index are unstable. Hence it may be said in addition that infinitely many nearby motions are of stable type and infinitely many other are of unstable type.

We may now start afresh with these new stable motions or with a stable motion corresponding to a known harmonic polygon with $n>2$, and discover further periodic motions by another application of the generalized theorem. In the next section we shall show how such a repetition leads to nearly periodic motions in the sense of Bohr, such as have not been proved hitherto to exist in dynamical problems.

Before doing so, however, it is of especial interest in the billiard ball problem to discuss the limiting periodic motions corresponding to the rolling of the ball around the table in the two possible senses. We propose to outline how an application of the generalized theorem leads to the conclusion that there exist infinitely many periodic motions uniformly near to these rolling motions, so that the corresponding harmonic polygons of $n$ sides lie in the immediate neighborhood of $C$. For this purpose it is essential to examine the explicit formulas given for $T$ in the case when $\theta$ is small. A direct computation leads to the result

$$
\left\{\begin{array}{l}
\theta_{1}=\theta-\frac{2 k^{\prime}}{3 k^{2}} \theta^{2}+l \theta^{3}+\cdots, \\
\varphi_{1}=\varphi+\frac{2}{k} \theta-\frac{4 k^{\prime}}{3 k^{3}} \theta^{2}+m \theta^{3}+\cdots,
\end{array}\right.
$$

where the function $k(\varphi)$ denotes the curvature of $C$ at the point with given $\varphi$ and where the functions $l, m, \ldots$ depend on $\varphi$ only. Proceeding entirely formally and 
replacing $\theta_{1}-\theta$ and $\varphi_{1}-\varphi$ by $d \theta$ and $d \varphi$ respectively, we obtain the approximate differential equation:

$$
\frac{d \theta}{d \varphi}=\frac{1}{3} \frac{k^{\prime}}{k} \theta
$$

which gives by integration

$$
\theta=\theta_{0} k^{\frac{1}{3}}(\varphi)
$$

Here $\theta_{0}$ is a value of $\theta$ for a point of curvature unity. This result indicates that, to a first approximation, the curve $\theta=\theta_{0} k^{\frac{1}{3}}(\varphi)$ near the inner boundary $\theta=0$ of the ring is nearly invariant under $T$, and can undoubtedly be modified slightly in higher order terms so as to be still more nearly invariant. Evidently the limiting motions formed by $C$ must be regarded as analogous to stable periodic motions on this account.

Also if the variable $n$ represents the number of iterations, we have the approximate differential equation

$$
\frac{d \varphi}{d n}=2 k^{-\frac{2}{3}}(\varphi) \theta_{0}
$$

whence by integration

$$
n=\frac{\mathrm{I}}{2 \theta_{0}} \int k^{\frac{2}{3}}(\varphi) d \varphi
$$

It follows that $\varphi$ will increase by more than $2 \pi$ along the approximate invariant curve if $\theta_{0} n$ exceeds $2 \pi k^{\frac{2}{3}}$ where $k$ denotes the maximum curvature of $C$.

It thus appears as highly probable that the generalized theorem is applicable to prove the existence of infinitely many periodic motions uniformly near to $C$, although I have not as yet worked through all of the details.

A number of further interesting geometric properties of nearly rolling motions of the billiard ball follow from these analytic facta. I will cite only one: if the ball is projected from a point $P$ of $C$ at a small angle $\theta$ with the tangent so as to return to $P$ after a large number of collisions with the boundary, then it will leave $P$ the second time at an angle which is $\theta$ to an arbitrarily high order in the infinitesimal $\theta$. 


\section{Existence of nearly periodic motions.}

Suppose now that we start with the infinite series of stable periodic motions, which are numerable in the general non-integrable problem. Let these be

$$
S_{1}, S_{2}, \ldots
$$

Choose a stable periodic motion $S_{k}$ near to $S_{1}$ but distinct from it (which exists in virtue of the generalized theorem as indicated above). Now choose an $S_{l}$ extraordinarily near to $S_{k}$ and distinct from $S_{2}$ also. In this way we can construct a series

$$
S_{k}, S_{l}, S_{m}, \ldots
$$

approaching a limit motion uniformly, call it $S$, and yet itself necessarily distinct from any periodic motion. Such a motion may be represented in the form

$$
p=\lim _{j=\infty} f_{j}(x), t=\lim _{j=\infty} g_{j}(\tau)
$$

where $p$ is any coordinate of the motion, where $\tau$ denotes a periodic variable of period $2 \pi$ along $S_{1}$ say, and where

$$
f_{j}(\tau) \quad \text { and } \quad g_{j}(\tau)-\frac{\tau_{j} \imath}{2 \pi l_{j}}
$$

are periodic functions of $\tau$ of period $2 \pi l_{j}$. The integer $l_{j}$ denotes the number of times the $j$ th motion of the sequence circulates about $S_{1}$ before reentering, and $\tau_{j}$ denotes its period. The convergence is uniform in $\boldsymbol{\tau}$.

It is clear from the manner of formation of these nearly periodic motions that they are non-denumerable, and constitute the class of uniform limits of periodic motions.

\section{The exceptional case.}

It has been stated that in order to apply the generalization of Poincaré's geometric theorem to the neighborhood of a stable periodic motion, either an invariant $e$ must not vanish, or at least one of an infinite set of similar constants must be different from zero. The exceptional case is that in which the period of the perturbed motion is independent of the constants of integration. 
The following example illustrates this category; there are only two stable periodic motions, and no other periodic motion whatsoever. In particular the example shows that the conjecture of Poincaré concerning the dense distribution of the periodic motions is not correct.

Imagine a particle of mass $m$ to move in a plane subject to a force derived from a potential energy:

$$
\frac{\mathrm{I}}{2} m\left(k^{2} x^{2}+l^{2} y^{2}\right)
$$

where $x$ and $y$ are the rectangular coordinates of the particle. The differential equations of motion are then

$$
\frac{d^{2} x}{d t^{2}}+k^{2} x=\mathrm{o}, \quad \frac{d^{2} y}{d t^{2}}+t^{2} y=\mathrm{o} .
$$

The particle moves so that its projections on the $x$ and $y$ axes describe harmonic motions about the origin of periods $\frac{2 \pi}{k}$ and $\frac{2 \pi}{l}$ respectively.

If we fix the energy constant $K^{2}$, we consider those solutions for which the relation

$$
\frac{m}{2}\left[\left(\frac{d x}{d t}\right)^{2}+\left(\frac{d y}{d t}\right)^{2}+k^{2} x^{2}+l^{2} y^{2}\right]=K^{2}
$$

holds. This equation shows that the motions all take place within an ellipse

$$
k^{2} x^{2}+l^{2} y^{2}=\frac{2 K^{2}}{m} .
$$

Through each point of the ellipse there is one and only one motion in a given direction, for this particular value of the energy constant. Thus we obtain a dynamical problem somewhat analogous to the billiard ball problem, although the velocity is now a known function of position and not a constant. Furthermore the problem is, of course, integrable with general solution

$$
x=A \cos k t+B \sin k t, y=C \cos l t+D \sin l t
$$

with the constants $A, B, C, D$ subject to the condition

$$
\frac{m}{2}\left[k^{2}\left(A^{2}+B^{2}\right)+l^{2}\left(C^{2}+D^{2}\right)\right]=K^{2}
$$


If we assume further that $k$ and $l$ are incommensurable with one another, obviously there can be no periodic motions except the two corresponding to the stable motions along the axes.

This problem can be reduced to a ring transformation, but such a reduction is not necessary for our purposes. We merely note that the transformation $T$ is essentially a rigid rotation of the ring through an angle incommensurable with $2 \pi$.

\section{Some further results.}

Associated with the instable periodic motions are the two analytic families of motions asymptotic to them. Poincaré pointed out that in the restricted problem of three bodies and for sufficiently small values of the parameters in-

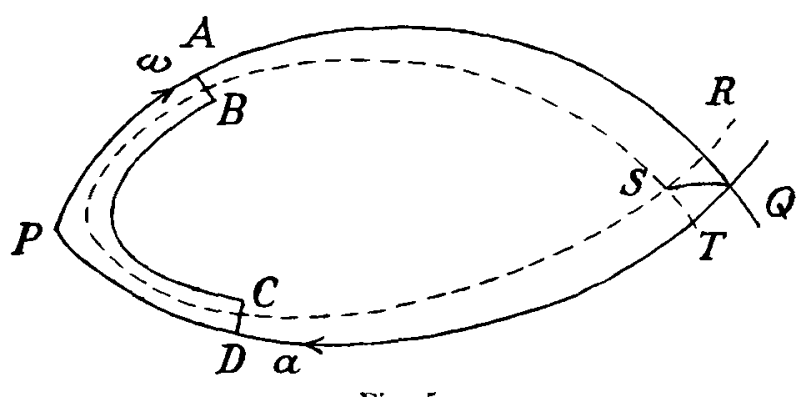

Fig. 5 .

volved, these families intersect one another infinitely often, giving rise to »homoclinic» motions, asymptotic to the given unstable periodic motion as time increases and as it decreases. It is certain that this intersection of the two families (in cases where they do not coincide identically) is a phenomenon of general occurrence, although $I$ have not as yet been able to treat certain exceptional cases.

I will prove here that every homoclinic motion is always in the immediate neighborhood of infinitely many periodic motions.

The fact just stated implies incidentally that the unstable periodic motion approached by the homoclinic motion lies in the immediate neighborhood of infinitely many other periodic motions, which cannot of course be entirely within a uniformly small neighborhood of this unstable motion.

In proving the result we shall confine attention to the case in which a ring transformation is at hand, although this is not really essential to the argument. In the figure the invariant point under $T$ it represented by $P$, with the asymptotic branches $\alpha$ and $\omega$ intersecting at $Q$ by hypothesis. Now it is a pro- 
perty of such an invariant point $P$ that the behavior of nearly points under iteration of $T$ is essentially the same as if $T$ were of the type

$$
u_{1}=p e^{c u v}, v_{1}=\frac{\mathrm{I}}{p} e^{-c u v} \quad(\mathrm{o}<p<\mathrm{I})
$$

In particular there will exist a family of invariant curves like $u v=$ constant, not perhaps analytic but possessing a high degree of regularity. The curvilinear pentagon $P A B C D$ represents a region near the invariant point analogous to a region:

$$
a>u>0, a>v>0 \quad u v \leqq c
$$

with $A B, C D$ analogous to $v=a, u=a$, with $P A, P D$ analogous to $u=0, v=0$, and with $B C$ analogous to $u v=c$. The dotted line within this pentagon represents one of the invariant curves.

It should be observed that as $c$ approaches zero, the curve $u v=c$ approaches the axes, and the number of iterations of $T$ during which a point on the curve remains within the pentagon becomes exceedingly large.

Choose now a value $c_{1}$ of $c$ so small that the curve $u v=c_{1}$ remains uniformly near the $\alpha$ and $\omega$ asymptotic branches until they intersect at $Q$. There is thus formed a curvilinear quadrilateral of intersection $Q R S T$ each point of which corresponds to a pair of parameter values $c^{\prime}, c^{\prime \prime}$, namely the parameter $c^{\prime}$ of the curve along the $\alpha$ branch passing through the point, and $e^{\prime \prime}$ the parameter of the curve along the $\omega$ branch passing through the same point. Thus there will be a curve $Q S$ (see the figure) for which $c^{\prime}=c^{\prime \prime}$, i.e. of points of intersection of one of the curves $u v=c$ constant with itself.

But the outermost segment of these curves $u v=c$ cut off by $Q S$ contains a certain number of segments, each of which is carried into the adjoining one by $T$, while a single partial segment may remain. Along another such segment with a smaller constant $c$, there will be many more such segments, at least if the constant is sufficiently small. Hence for some intermediate value of the constant there will be an exact integral multiple $k$ of such segments, and the point of $Q S$ lying on the curve will be carried into itself by the $k$ th iterate of $T$.

It is interesting to note that the maximum-minimum method (for the case of the billiard ball problem at least) gives some information of the same sort.

Suppose that the longest chord of the convex curve $C$ has a length $l$, and suppose that the harmonic polygon of $q$ sides ( $q$ odd) be sought which has maximum 48-26404. Acta mathematica. 50. Imprimé le 28 octobre 1927. 
length. This maximum length exceeds $(q-1) l$ because a polygon may be chosen made up of the longest chord taken $q-1$ times together with one side of length zero. The length cannot be as much as $q l$, since $l$ is the length of the longest chord. Nor can the maximizing polygon lie near the longest chord throughout its length, or it would, of course, have an even number of sides. On the contrary, one side of it must be at least as long as $\frac{(q-1) l}{q}$, and so will be very near to the longest chord if $q$ is large. Consequently there are infinitely many harmonic polygons lying in general very near to the longest chord, but leaving its immediate vicinity at least once. A refinement of this argument leads to the conclusion that there exist motions homoclinic to the fundamental unstable periodic motion also. It is not obvious that the maximum-minimum method will lead to like conclusions for the more complicated harmonic polygons corresponding to unstable periodic motions.

The successful application of the above method for the derivation of additional periodic motions of dynamical systems with two degrees of freedom requires the existence of motions homoclinic to unstable periodic motions.

It will be noted that the requirement of an invariant area integral has not entered into the above reasoning, so that the criterion may be applied to differential systems, which are not associated with a dynamical problem.

\section{The totality of periodic motions.}

Thus if a dynamical system with two degrees admits of a single stable periodic motion of non-exceptional type, it admits of infinitely many other stable periodic motions in its immediate vicinity. Consequently the totality of such stable periodie motions forms a set dense in itself, with nearly periodic limiting motions of the Bohr type. Each stable periodic motion has also infinitely many other unstable periodic motions in its vicinity, which in turn will be approached (but not uniformly) by infinitely many periodic motions, at least if certain homoclinic motions exist.

It still remains an open question as to whether or not the periodic motions are densely distributed throughout the possible motions. This cannot be true 
unconditionally, as the example given above makes clear. On the other hand I have shown that the periodic motions together with those asymptotic to them are everywhere dense in the transitive case. ${ }^{1}$

${ }^{1}$ See my earlier Acta article. A system is transitive if motions can be found passing from nearly one assigned state to nearly any other arbitrarily assigned state. This property is probably satisfied "in general" by non-integrable dynamical systems. 